\title{
NANOCONE STRUCTURES WITH LIMITED INTERSPACE GROWN BY MOVPE
}

\author{
J. Novák ${ }^{a}$, P. Eliáš ${ }^{\text {a }}$, S. Hasenöhrl a ${ }^{\text {, A. Laurenčíková }}{ }^{a}$, P. Urbancová ${ }^{b}$, and D. Pudiš ${ }^{b}$ \\ ${ }^{a}$ Institute of Electrical Engineering SAS, Dubravská 9, 84104 Bratislava, Slovakia \\ ${ }^{\mathrm{b}}$ Department of Physics, University of Žilina, Univerzitná 1, 01008 Žilina, Slovakia \\ Email: jozef.novak@savba.sk
}

Received 10 June 2019; revised 16 September 2019; accepted 16 September 2019

\begin{abstract}
Reduction and limitation of free spaces between the gallium phosphide nanocones was studied. A set of nanocone samples was grown by metal organic vapour phase epitaxy (MOVPE) in the temperature range between 610 and $690^{\circ} \mathrm{C}$. Our results showed that by an appropriate combination of a high density of gold seeds and an optimized growth temperature it was possible to obtain a nanostructured surface with very limited free spaces between the nanocones. A combination of lateral and vertical growth rates regulated by the selection of growth temperature played a very important role in the nanocone hexagonal base enlargement, which helped to minimize spaces between the cones. The limitation of free space between the nanocones increased a probability of edge creation that is very helpful for the successful growth of 2D materials.
\end{abstract}

Keywords: nanocone, epitaxy, gold seed, shell layer

\section{Introduction}

Semiconductor nanowires have recently emerged as a new class of materials with a significant potential to reveal new fundamental physical effects and to propel new applications in quantum electronic, optoelectronic and photonic devices. This giant application potential is partially hindered by their brittleness and fragility. Transfer from nanowires to more robust nanocones can circumvent the undesirable properties of nanowires. Recent advances in nanotechnology have enabled the well-controlled bottom-up fabrication of nanocones (NCs). This progress has opened a large space for the preparation of various heterojunctions between advanced NC structures and progressive modern materials. It was recently shown that edges of nanostructures induce a high quality epitaxial growth of $\mathrm{MoS}_{2}$ in spite of a large lat- tice mismatch and layered structure. Epitaxy offers a controllable method for the preparation of lateral heterojunctions with atomically sharp interfaces [1]. Additionally, the spray pyrolysis of a $\mathrm{ZnCl}_{2}$ solution was used to grow symmetrically faceted $\mathrm{ZnO}$ nanorods on highly oriented pyrolytic graphite substrates. It was shown that such crystallites nucleated preferably at the multilayer graphene sheet edges. This was attributed to a better thermal stability and larger surface density of adsorbed oxygen species at the edges. The naturally occurring edge-joint between a multilayer graphene sheet and a semiconductor nanorod was demonstrated for the first time [2].

The theoretical and experimental studies have revealed the new finding in the substantial improvements of both optical and electrical properties based on the geometrically designed $3 \mathrm{D}$-graphene $\mathrm{MoS}_{2}$ structures [3]. The structures 
exhibited excellent light absorption (between 300 and $800 \mathrm{~nm}$ ), which was attributed (1) to the favourable energy band for the efficient charge transfer between the electronically inter-connected graphene and $\mathrm{MoS}_{2}$, and (2) to the orientation of the $\mathrm{MoS}_{2}$ crystal face array. As regards the charge transport, the edge-rich $\mathrm{MoS}_{2}$ grown on the edgeoriented 3D graphene glass can achieve an optimized charge transport along the $2 \mathrm{D}$ vector plane from the $\mathrm{MoS}_{2}$ layers to the graphene. Consequently, such hybrid nanostructures exhibited excellent performance as an effective photocatalyst for hydrogen generation from photocatalytic water splitting [4]. These and many other studies showed that edges play an important role for the deposition of thin $\mathrm{MoS}_{2}$ layers and structures.

In our previous studies we have shown that $\mathrm{GaP}$ nanocones can be used for the well-defined deposition of other attractive materials, such as $\mathrm{ZnO}$ or $\mathrm{MoS}_{2}$ that were proposed for advanced applications [5, 6]. Quality of some deposited layers can be improved by growth on structures with an increased density of growth edges for preferential nucleation. In this paper we present the preparation of a $\mathrm{GaP}$ substrate structured with closely grown $\mathrm{GaP}$ nanocones. Such a substrate exhibited a large density of edges on $\mathrm{GaP}$ nanostructures and that reduced to a minimum the original surface area of the $\mathrm{GaP}$ substrate.

\section{Experiment}

Zinc-doped gallium phosphide ( $\mathrm{GaP}$ ) nanocones were prepared by metal organic vapour phase epitaxy (MOVPE) on GaP[111]B substrates. The gallium phosphide nanocones that grew on such substrates had [111]-oriented cross-sections of hexagonal symmetry. It was possible to grow very dense nanocone assemblages that almost eliminated the original [111]B-oriented substrate surface. This provided numerous edges for the subsequent preferential edge growth of $\mathrm{MoS}_{2}$. The GaP nanocones were prepared by a vapour-liquid-solid (VLS) technique using gold particles as seeds that self-assembled from very thin $(<\sim \mathrm{nm}) \mathrm{Au}$ films. The films were deposited on the GaP substrate by evaporation. The seeds acted as a catalyst for the growth of GaP NCs. The films differed in thicknesses (from 1 to $0.1 \mathrm{~nm}$ ), and $\mathrm{Au}$ seeds with various diameters were formed. Prior to the growth of NCs, Au covered samples were inserted into the MOVPE chamber of an AIX 200 MOVPE low-pressure reactor, heated at $650^{\circ} \mathrm{C}$, and maintained at this temperature for $10 \mathrm{~min}$ under $\mathrm{H}_{2}+\mathrm{PH}_{3}$ flow. The role of this step was to desorb surface oxides and to allow for the formation and alloying of the seeds. Next, the growth of NCs was performed at different growth temperatures between 500 and $690^{\circ} \mathrm{C}$ and a pressure of 100 mbar. All nanostructures were doped by zinc to assure a $p$-type conductivity [四.

\section{Results}

\subsection{Density of seeds}

At first we studied the formation of gold seeds from very thin Au layers during the growth process. Only the alloying phase of the VLS process was performed. In these experiments gold layers with an equivalent thickness of $0.3,0.5,0.9$ and $1 \mathrm{~nm}$ were evaporated on the GaP surface and alloyed during the first phase of the MOVPE growth process. The self-assembled $\mathrm{Au}$ seeds exhibited very similar size distributions regardless of the thickness of the Au layer, but Au seed inter-distance distributions showed slightly longer inter-distances for thinner Au layers (lower densities). The seed density was estimated to be about $300,270,200$ and 100 at $500 \times 500 \mathrm{~nm}^{2}$. The average seed diameter was close to $30 \mathrm{~nm}$. An example of the wafer surface covered by gold seeds after the alloying phase is presented in Fig. 1. Two of seed distributions, mentioned above, are compared in the figure. The distribution of the gold seeds was uniform over the whole wafer surface.

\subsection{Properties of nanostructures}

The growth temperature increase led to a substantial change of the nanostructure shape, starting from nearly cylindrical $\left(T_{\mathrm{g}}=500^{\circ} \mathrm{C}\right)$ nanowires to tapered nanocones $\left(T_{\mathrm{g}}\right.$ between 610 and $650^{\circ} \mathrm{C}$ ) up to biconical shapes for $T_{\mathrm{g}}=690^{\circ} \mathrm{C}$. The shape of nanostructures grown at higher temperatures is typically tapered (see Fig. 2). The cross-section at the base is hexagonal (it corresponds to substrate $<111>$ B substrate orientation), and consequently the nanocones offer a large adsorption surface for the deposition of shell material. Sidewalls of 


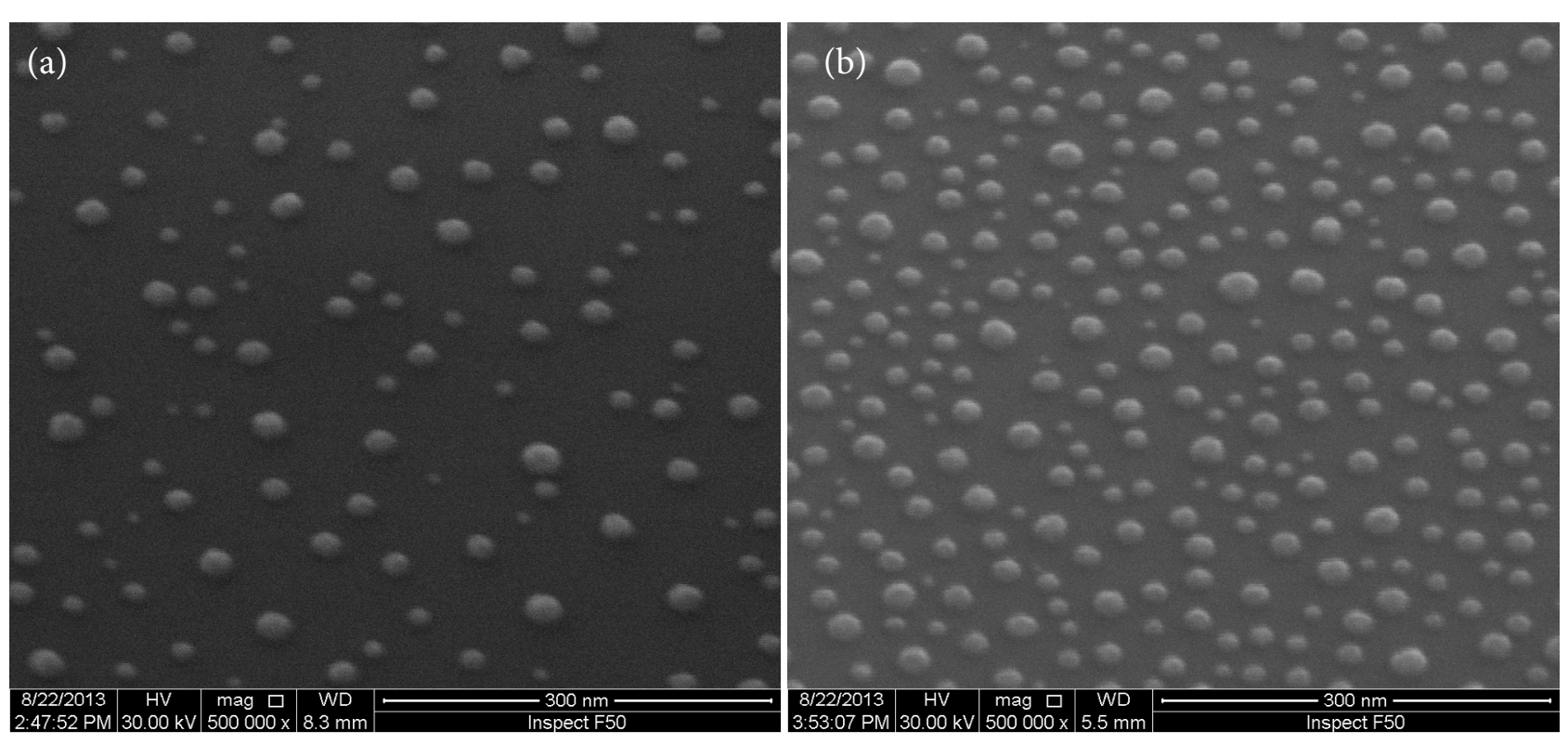

Fig. 1. SEM image of the GaP(111)B substrate strewn randomly with Au seeds deposited from a $0.3 \mathrm{~nm}$ thick Au layer (a) and $1.0 \mathrm{~nm}$ thick Au layer (b), and alloyed at $650^{\circ} \mathrm{C}$.
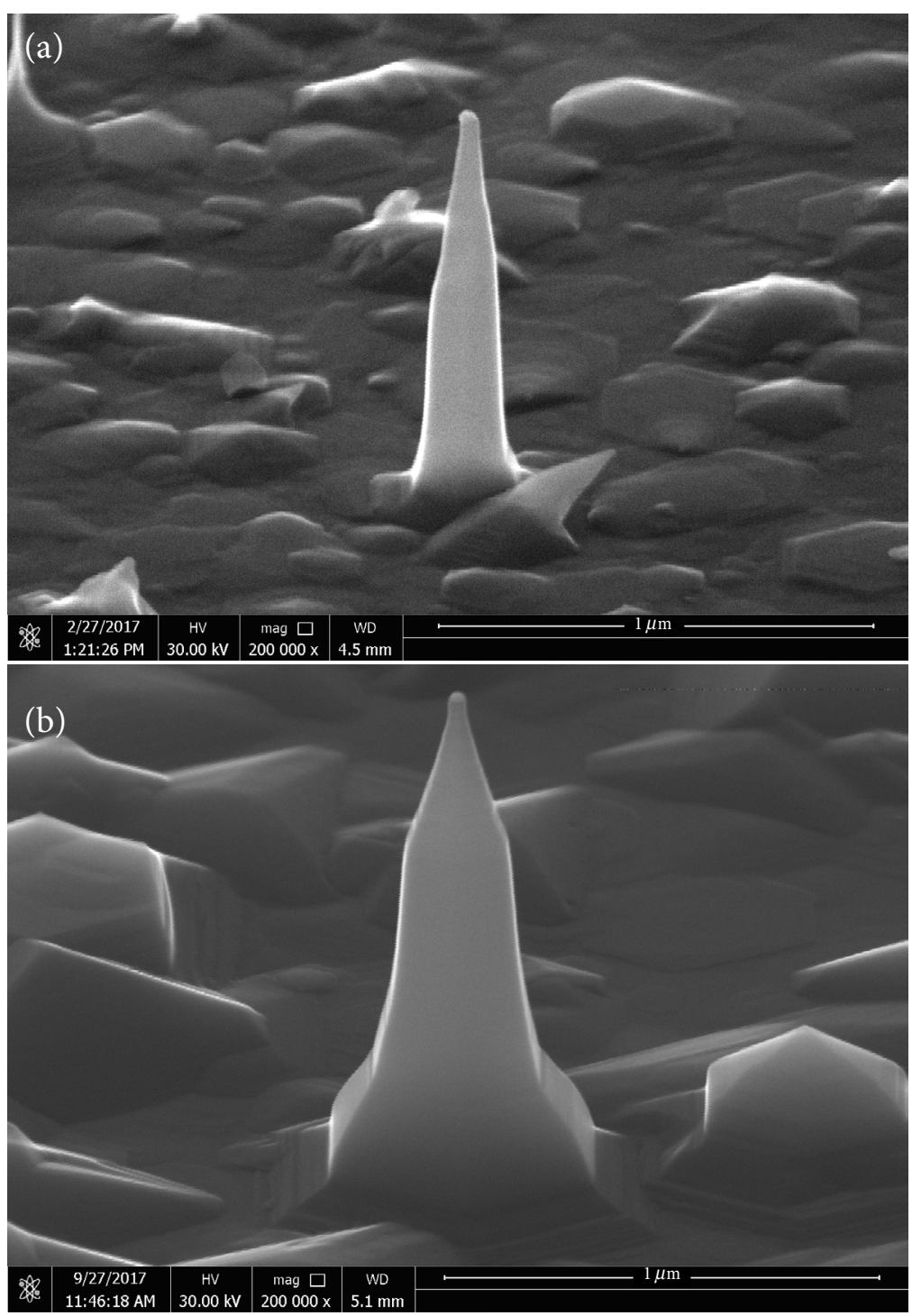

Fig. 2. SEM images of GaP nanocones grown at various growth temperatures: $630^{\circ} \mathrm{C}(\mathrm{a}), 670^{\circ} \mathrm{C}(\mathrm{b})$. The images present a substantial difference in the shape of the nanocones. 
NCs grown at temperatures higher than $650^{\circ} \mathrm{C}$ are tapered and cranked with a turning line in the bottom part. The size of such NCs depends more or less linearly on the growth time.

The relation between the thickness of the gold layer evaporated onto the substrate surface and the final density of seeds allowed us to control the density of nanocones and their mutual distance. Application of some experimental experience and strict precision allowed us to prepare structures with a very small inter-particle distance with a high degree of reproducibility. In our initial experiment large-density $\mathrm{GaP}$ nanocone structures were grown at $650^{\circ} \mathrm{C}$ temperature at $\mathrm{Au}$ seeds prepared from a $0.3 \mathrm{~nm}$ thick Au layer. We suppose that a combination of vertical and lateral growth together with the starting density of $\mathrm{Au}$ seeds should deliver the optimal large density of resulting edges. Figure 3 indicates a very successful achievement of this task.

\subsection{Optical properties}

We studied optical properties of the samples grown by the technique described above. The backside of all samples used for the optical measurement was mechanically-chemically polished. Before polishing all samples were glued to a sample holder using a thermally controlled phthalate resin. The samples were polished and then detached from the hold- er as they were slightly heated. The samples were cleaned in acetone to remove the glue. Damage done to the samples during the polishing process was inspected by SEM. An Ocean Optics spectrometer was used to determine angular dependences of the reflectance and transmission of the NC structures. As the reference structure, a $\mathrm{GaP}$ substrate with a polished backside was measured and compared with a $\mathrm{GaP}$ substrate covered with $\mathrm{GaP}$ NCs. This comparison was done for each wavelength at normal incidence. The reflectance was measured for different angles of the reflection and compared to the reflection of the reference planar $\mathrm{GaP}$ substrate. The reference reflection value of the GaP substrate was measured for each reflection angle. Figure 4 shows that the NC samples exhibited two different types of reflectance behaviour. For wavelengths between 400 and $550 \mathrm{~nm}$, the reflectivity was relatively high, and it strongly depended on the angle of measurement. In this wavelength interval, the NC structure behaved as a special mirror-like nanopatterned structure. Here the optical properties are determined by a combination of the size effect and mirror-like properties of the (sixfold) sidewall triangular facets of nanopyramids. The reflectivity at wavelengths above $550 \mathrm{~nm}$ was given by the optical properties and absorption/reflection behaviour of $\mathrm{GaP}$ as semiconductor material with a band gap of $2.2 \mathrm{eV}$ and the relevant absorption/reflection behaviour of a pyramidal structure.

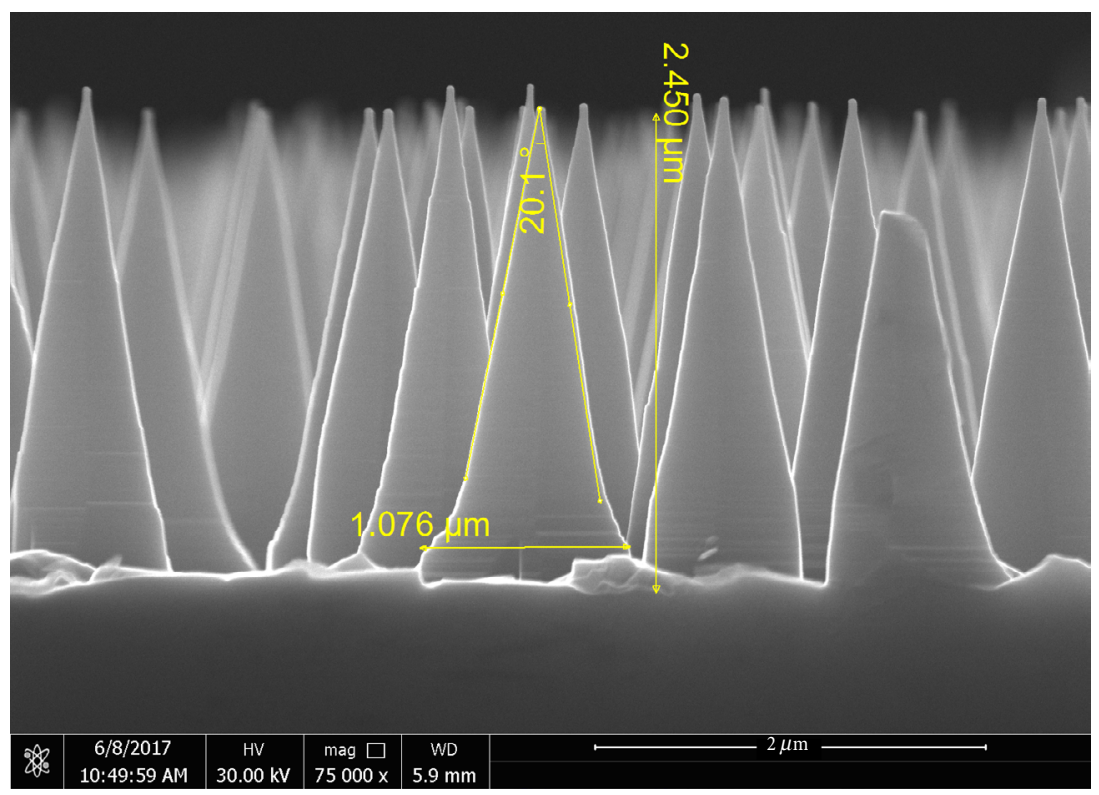

Fig. 3. SEM sideview of a forest of $\mathrm{GaP}$ nanocones prepared from $\mathrm{Au}$ seeds at $650^{\circ} \mathrm{C}$ growth temperature. 


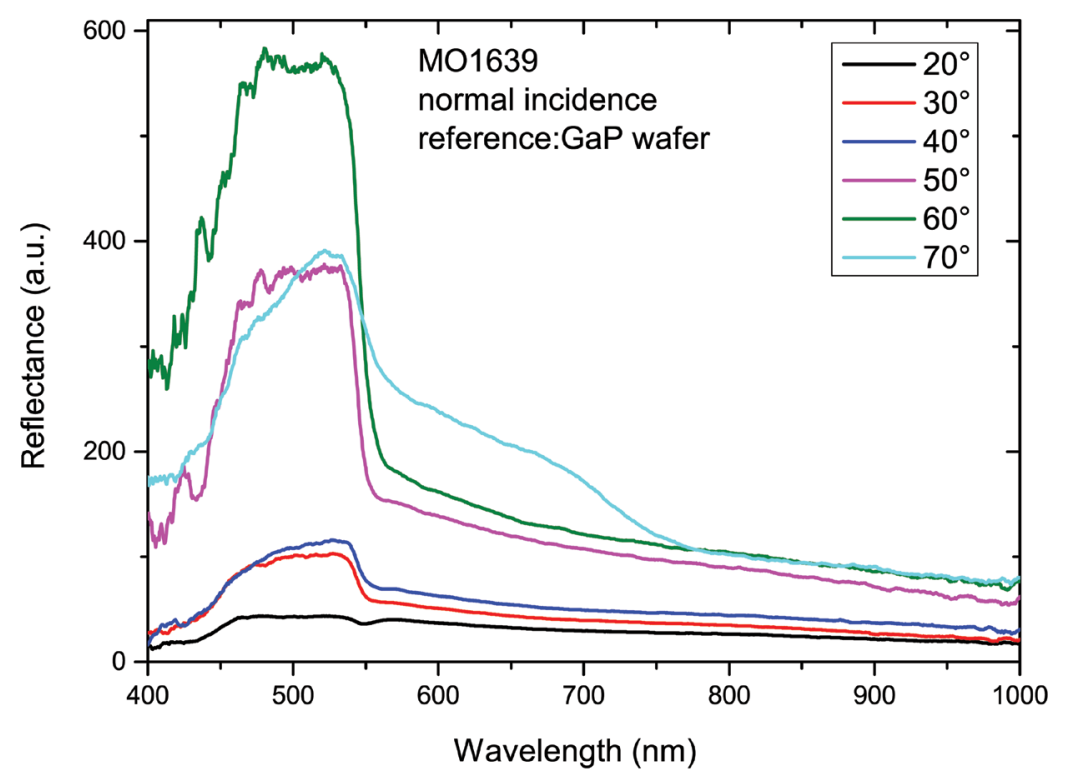

Fig. 4. Reflectance of a GaP nanocone structure measured at normally incident light at various reflection angles.

Figure 5 shows a spectral dependence of the transmission of GaP NCs compared with that of the reference planar $\mathrm{GaP}$ substrate for wavelengths between 400 and $1000 \mathrm{~nm}$. This figure shows that the reference wafer exhibits standard transmission properties of the semiconductor material, i.e. a moderately increasing transmission starting from the band gap value. However, the transmission of the NC structure was significantly lower. Its transmission in relation to the wavelength also increased, but the value was generally lower. This transmission decrease is caused by reflection of incident power from nanocone sidewall facets, as demonstrated in Fig. 4 .

\section{Conclusions}

The MOVPE growth of nano-patterned structures with a high density of nanocones with the aim to minimize intercone distances was studied. Our

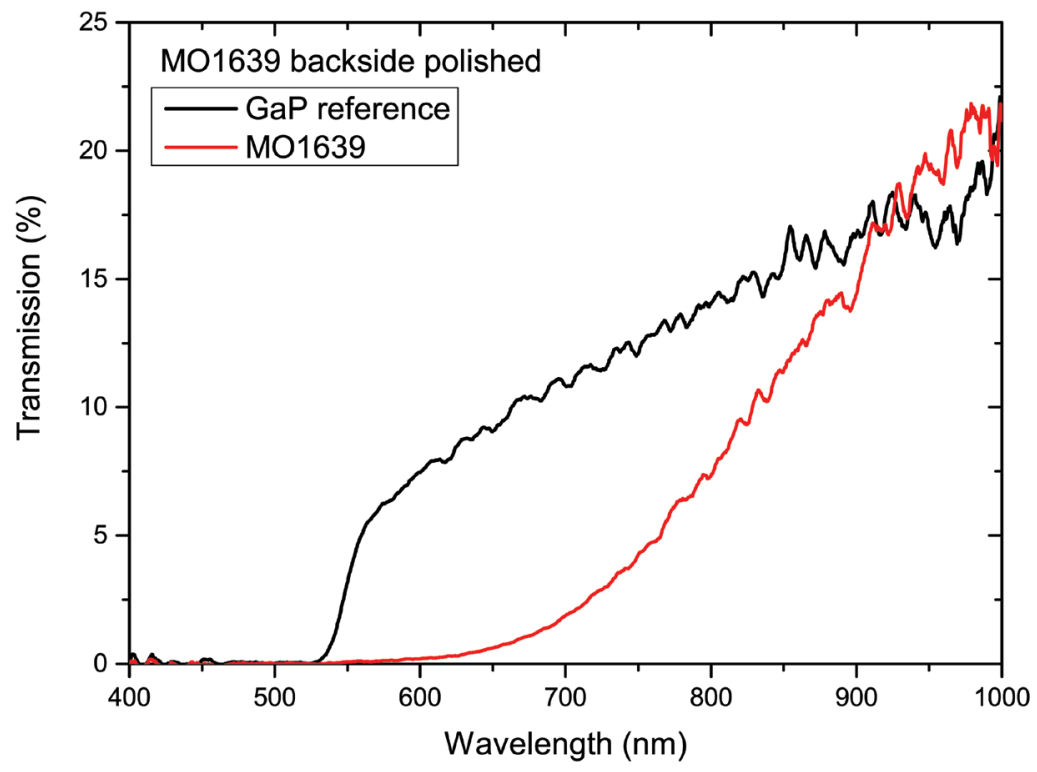

Fig. 5. Transmission of a GaP nanocone structure compared with that of the $\mathrm{GaP}$ reference wafer. 
experiments showed that by an appropriate combination of a high density of seeds and an optimized growth temperature it was possible to obtain a nanostructured surface with very limited free spaces between the nanocones. A combination of lateral and vertical growth rates regulated by the selection of growth temperature played a very important role in the the nanocone hexagonal base enlargement, which helped to minimize spaces between the cones. The most suitable and balanced option was achieved at $650^{\circ} \mathrm{C}$ growth temperature and $\mathrm{Au}$ seeds prepared from a $0.3 \mathrm{~nm}$ thick Au layer. At this combination of growth conditions, the nanocone sidewalls were flat and sidewall edges were well defined by facet edges.

\section{Acknowledgements}

This research was supported by the Science and Technology Assistance Agency under Grant No. APVV-14-0297 together with Grant APVV16-0129 and by VEGA Project No. 2/0104/17.

\section{References}

[1] M.Y. Li, Y. Shi, Ch.Ch. Cheng, L.S. Lu, Y.Ch. Lin, H.L. Tang, M.L. Tsai, Ch.W. Chu, K.H. Wei, J.H. He, W.H. Chang, K. Suenaga, and L.J. Li, Epitaxial growth of a monolayer $\mathrm{WSe}_{2}-\mathrm{MoS}_{2}$ lateral p-n junction with an atomically sharp interface, Science 349, 524-528 (2015).
[2] H.U. Kim, M. Kim, Y. Jin, Y. Hyeon, K.S. Kim, B.S. An, Ch.W. Yang, V. Kanad, J.Y. Moon, G.Y. Yeom, D. Whang, J.H. Lee, and T. Kim, Low-temperature wafer-scale growth of $\mathrm{MoS}_{2}-$ graphene heterostructures, Appl. Surf. Sci. 470, 129-134 (2019).

[3] F. Hossein-Babaei and M. Akbari-Saatlu, Growth of $\mathrm{ZnO}$ nanorods on the surface and edges of a multilayer graphene sheet, Scr. Mater. 139, 7782 (2017).

[4] X. Lia, Sh. Guoa, W. Li, X. Renc, J. Sud, Q. Song, and A.J. Sobridob, Edge-rich $\mathrm{MoS}_{2}$ grown on edge-oriented three-dimensional graphene glass for high-performance hydrogen evolution, Nano Energy 57, 388-397 (2019).

[5] J. Novák, A. Laurenčíková, P. Eliáš, S. Hasenöhrl, M. Sojková, E. Dobrocka, J. Kováč Jr., J. Kováč, J. Duurišová, and D. Pudiš, Nanorods and nanocones for advanced sensor applications, Appl. Surf. Sci. 461, 61-65 (2018).

[6] A. Laurenčíková, P. Eliáš, S. Hasenöhrl, J. Kováč Jr., and J. Novák, GaP nanocones covered by silver nanoparticles for surface enhanced Raman spectroscopy, Appl. Surf. Sci. 461, 149-153 (2018).

[7] S. Hasenöhrl, P. Eliáš, J. Šoltýs, R. Stoklas, A. Laurenčíková, and J. Novák, Zinc-doped gallium phosphide nanowires for photovoltaic structures, Appl. Surf. Sci. 269, 72-76 (2013).

\title{
KŪGIŠKI TANKŪS NANODARINIAI, IŠAUGINTI METALORGANINĖS GARŲ FAZĖS EPITAKSIJOS BŪDU
}

\author{
J. Novák ${ }^{\text {a }}$ P. Eliáš a ${ }^{\text {, S. Hasenöhrl a }}$, A. Laurenčíková a ${ }^{\text {, P. Urbancová }}{ }^{\text {b }}$ D. Pudiš ${ }^{b}$ \\ a Slovakijos mokslu akademijos Elektros inžinerijos institutas, Bratislava, Slovakija \\ b Žilinos universiteto Fizikos fakultetas, Žilina, Slovakija
}

